\title{
Heartbeat: Improving acute myocardial infarction outcomes
} in women

doi:10.1136/heartjnl-2019-315000

Catherine $\mathrm{M} \mathrm{Otto}^{\circ}$

In this issue of Heart, Wilkinson and colleagues ${ }^{1}$ report sex differences in guideline-indicated care for acute myocardial infarction (AMI) from a nationwide cohort study of over 690 thousand AMI hospitalisations in England and Wales. Women comprised about 35\% of the cohort and were older than men (mean age 77 vs 67 years) but less often had ST-elevation myocardial infarction (STEMI, 34\% vs 43\%). In addition, women less often received timely reperfusion therapy for STEMI, timely coronary angiography for non-STEMI, treatment with dual antiplatelet therapy or secondary prevention medications (figure 1). Median 30 day risk score adjusted mortality was higher in women compared with men (median: $5.2 \%$ (IQR 1.8\%-13.1\%) vs $2.3 \%$ (IQR $0.8 \%-7.1 \%$ ), $\mathrm{p}<0.001$ ) and the authors estimate that 8243 (95\% CI 8111 to 8375 ) deaths among women could have been prevented if quality indicators in women had been equivalent to those observed in men.

In an editorial, Wei et $a l^{2}$ point out that improving outcomes after AMI in women requires an increased understanding of both the biological sex differences and gender bias in medical care. Biological differences include the higher prevalence of myocardial infarction with non-obstructive coronary arteries (MINOCA) in women with various mechanisms leading to AMI including coronary vasospasm, embolism, spontaneous coronary dissection and Takotsubo cardiomyopathy. These biological differences are then confounded by gender bias in treatment. Protocols focused on improving recognition of AMI, rapid transfer to a centre capable of percutaneous intervention and checklists to ensure optimal medical therapy can improve outcomes in both men and women (figure 2). They conclude: 'It is clear from this new study and work of others that protocols for the delivery of recommended AMI treatments for women can reduce the sex-AMI mortality gap. Accordingly, the question

Correspondence to Professor Catherine M Otto, Division of Cardiology, University of Washington, Seattle WA 98195, USA; cmotto@uw.edu

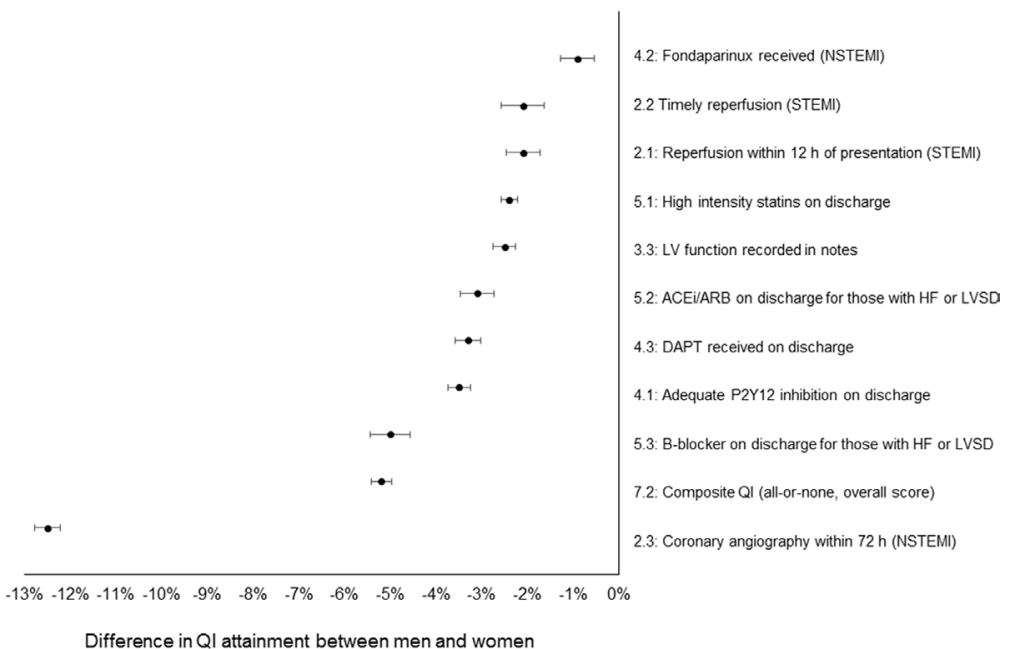

Figure 1 Mean difference in QI attainment between men and women. ACEi, ACE inhibitor; ARB angiotensin receptor blocker; DAPT, dual antiplatelet therapy; HF, heart failure; LV, left ventricular; LVSD, left ventricular systolic dysfunction; NSTEMI, non-STEMI; QI, quality indicator; STEMI, STelevation myocardial infarction. is: do we have the will to improve CVD outcomes for women?'

In a study from New Zealand ${ }^{3}$, MINOCA accounted for about $11 \%$ of patients in a nationwide cohort study of over 8000 AMI patients. In those with obstructive coronary artery disease, all-cause mortality at 2 years was $7.9 \%$ compared with $4.9 \%$ in those with MINOCA. The cause of death was split relatively evenly between cardiac and non-cardiac causes in those with obstructive coronary disease. In contrast, most deaths in patients with MINOCA were due to non-cardiac causes (figure 3 ).

The link between the New Zealand study on MINOCA ${ }^{3}$ and the UK based study $^{1}$ on sex differences in AMI treatment is evident in the editorial by Mehta

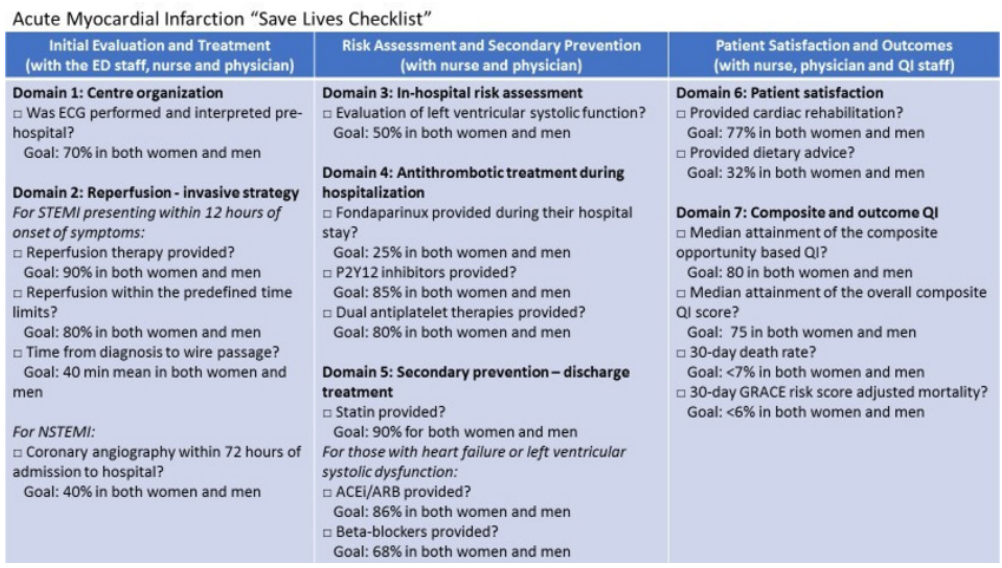

Figure 2 AMI 'Save Lives Checklist' from six quality indicators of the European Society of Cardiology Acute Cardiovascular Care Association suite of Quality Indicators (QIs) for AMI. The checklist is not intended to be comprehensive. Additions and modifications to fit local practice are encouraged. The initial goals reflect the metrics achieved in men and can be updated for further improvement with measured progress over time. ACEi, ACE inhibitor; AMI, acute myocardial infarction; $A R B$, angiotensin receptor blocker; ED, emergency department. 


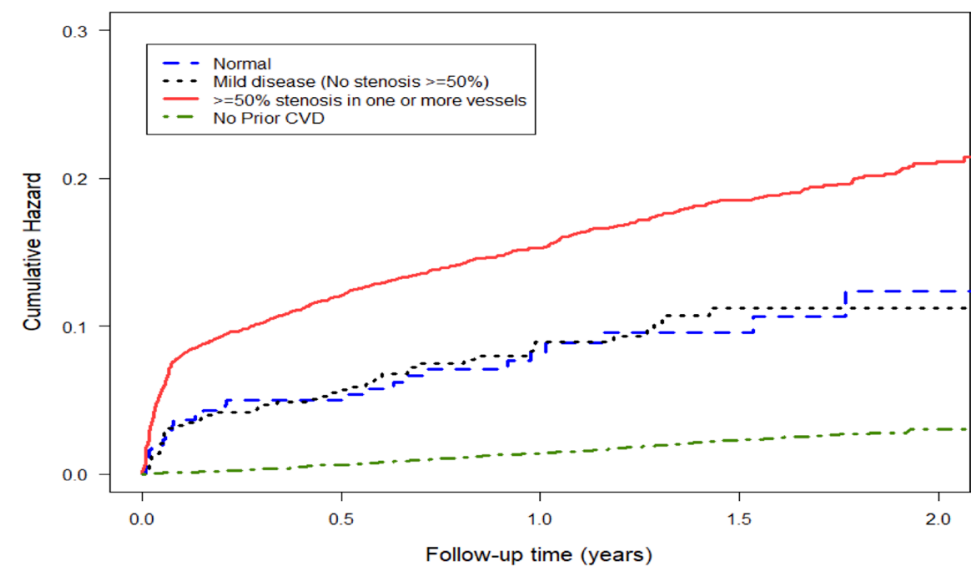

$\begin{array}{lc}\text { Patients at risk } & \\ \text { Follow-up time } & 0 \\ \text { Normal } & 308 \\ \text { Mild } & 589 \\ \text { 250\% stenosis } & 7408 \\ \text { No Prior CVD } & 8305\end{array}$

0.5
257
486
5567
7686

1.0
163
305
3553
6432

1.5
97
166
1928
5103

2.0
26
39
536
1178

Figure 3 Kaplan-Meier analysis of all-cause mortality or non-fatal myocardial infarction for patients with and without obstructive coronary artery disease and for patients without prior cardiovascular disease (CVD).

and Beltrame ${ }^{4}$ in which they raise the question: 'Why do young women predominate in MINOCA population? Even in the settings of stable angina and ischaemic heart disease, women are more likely to have the finding of no obstructive CAD on angiography.' They go on to answer that: 'Coronary microvascular dysfunction from either abnormal vasodilatory capacity or from microvascular spasm is highly prevalent in this group. Vascular disorders such as spontaneous coronary artery dissection (SCAD) and fibromuscular dysplasia that lead to MI are also more prevalent in younger women who often do not have traditional risk factors such as hyperlipidaemia or diabetes.' Furthermore: 'Whether patients with MINOCA should receive the same

treatment regimen for secondary prevention over longer term as used for those with obstructive CAD is unknown. Given the adverse prognosis in MINOCA, close physician follow-up is required to ensure optimal risk factor management and adequate angina control.'

In patients with adult congenital heart disease, standard cardiac treatments may be associated with complications that differ from the general cardiology population. For example, Egbe et $\mathrm{ll}^{5}$ found that in tetralogy of Fallot (ToF) patients with an implantable cardiac electronic device, complications occurred in 20\% including lead failure, lead recall, device infection and thrombus. The annualised rate of appropriate shocks was $5.7 \%$ compared with an annual inappropriate shock rate of $6.2 \%$. Deen and

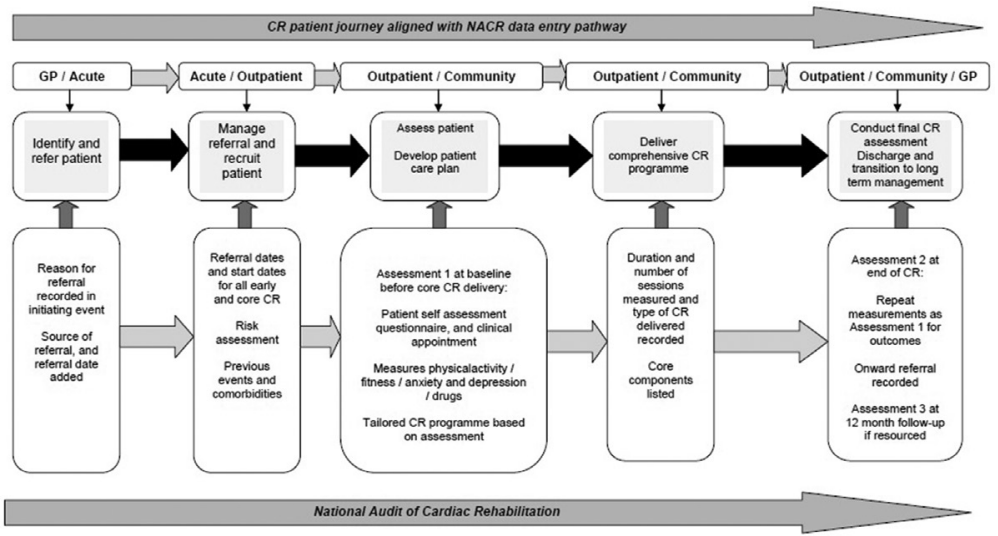

Figure 4 Cardiac rehabilitation patient pathway aligned with NACR data entry pathway. CR, cardiac rehabilitation; GP, general practitioner; NACR, National Audit of Cardiac Rehabilitation.
Prutkin $^{6}$ put this data in context, noting that arrhythmias are common in adult patients with a repaired ToF and that there is an increased incidence of atrial fibrillation and ventricular arrhythmias after age 45 years. Although risk stratification for primary prevention of sudden cardiac death in ToF patients is imperfect, 'the recent American Heart Association/American College of Cardiology Guideline for the Management of Adults with Congenital Heart Disease gives a IIa recommendation for a primary prevention ICD in those patients with multiple risk factors for sudden cardiac death (left ventricular systolic or diastolic dysfunction, non-sustained ventricular tachycardia, QRS duration $>180 \mathrm{~ms}$ or extensive myocardial fibrosis on cardiac MRI).'

The Education in Heart article in this issue provides a summary of how imaging can be used to as a marker of drug efficacy and disease activity. ${ }^{7}$ In addition, there is an excellent review article ${ }^{8}$ summarising the standards and core components for cardiovascular disease prevention and rehabilitation (figure 4).

\section{Competing interests None declared.}

Patient consent for publication Not required.

Provenance and peer review Commissioned; internally peer reviewed.

(C) Author(s) (or their employer(s)) 2019. No commercial re-use. See rights and permissions. Published by BMJ.

\section{Check for updates}

To cite Otto CM. Heart 2019;105:501-502.

Heart 2019;105:501-502.

doi:10.1136/heartjnl-2019-315000

\section{REFERENCES}

1 Wilkinson C, Bebb O, Dondo TB, et al. Sex differences in quality indicator attainment for myocardial infarction: a nationwide cohort study. Heart 2019;105:516-23.

2 Wei J, Henry TD, Bairey Merz CN. Biology and bias: do we have the will to improve cardiovascular disease outcomes for women? Heart 2019;105:503-5.

3 Williams MJA, Barr PR, Lee M, et al. Outcome after myocardial infarction without obstructive coronary artery disease. Heart 2019;105:524-30.

4 Mehta PK, Beltrame JF. Myocardial infarction with nonobstructive coronary arteries: a humbling diagnosis in 2018. Heart 2019;105:506-7.

5 Egbe AC, Miranda WR, Madhavan M, et al. Cardiac implantable electronic devices in adults with tetralogy of Fallot. Heart 2019;105:538-44.

6 Deen JF, Prutkin JM. Shock to the heart: cardiac implantable devices' bad name in adults with tetralogy of Fallot. Heart 2019;105:508-9.

7 Tarkin JM, Dweck MR, Rudd JHF. Imaging as a surrogate marker of drug efficacy in cardiovascular disease. Heart 2019;105:566-77.

8 Cowie A, Buckley J, Doherty P, et al. Standards and core components for cardiovascular disease prevention and rehabilitation. Heart 2019;105:510-5. 